RTD time was $<31$ days in $57 / 112$ cases $(50.8 \%)$. $31.6 \%$ of these were Stage I-IIIA, compared with $54.5 \%$ Stage I-IIIA when RTD was $>31$ days.

RTT time was $<62$ days in 59/112 cases (52.7\%). 25.4\% of these were Stage I-IIIA, compared with $62.3 \%$ Stage I-IIIA when RTT was $>62$ days.

RTT time was $<62$ days in $15 / 48$ (31.3\%) Stage I-IIIA patients and $<62$ days in 44/64 (68.8\%) patients with Stage IIIB-IV.

Conclusions Despite few CWT breachers, RTT times were frequently $>62$ days suggesting pathway adjustments have a major impact. Patients with earlier stage disease, and the most to lose from diagnostic delay had longer diagnostic journeys. The survival disadvantage of short pathways likely reflects stage mix. Pathway redesign to accelerate the complex diagnostics needed for radically-treatable disease should be considered. CWT adjustments may have unintentionally clouded this issue.

\section{P85 VIRTUAL LUNG CANCER CLINIC: EARLY EXPERIENCE AND FEASIBILITY}

JF Faccenda, LD Calvert, SO Brij. Peterborough City Hospital, Peterborough, UK

\subsection{6/thoraxinl-2016-209333.228}

Background With increased public awareness, cough campaigns and incidental nodules on computed tomography (CT), referrals on a Lung Cancer Pathway (LC) have risen significantly. Safe and effective methods to transfer patients to Respiratory Pathways (RP) are essential.

Aims to evaluate a chose and book, virtual Lung Cancer Clinic (VLCC) to facilitate non-face-to-face "blind" rapid patient assessment, next investigation and appropriate out-patient review.

Methods A retrospective review of all referrals during the period March-May 2016 was undertaken to assess whether blind clinical decision-making at point of referral was sufficient to plan ongoing management.

Results 60 referrals were reviewed in VLCC by a Lung Cancer Consultant Physician (average time from referral 2 days, range 0-4 days) as their first 2 week wait appointment. 17 (28\%) patients had a final diagnosis of Lung Cancer (histological 12, radiological 5).

Only 29/60 (48\%) were of an acceptable quality for blind decision making. 16 (27\%) referrals did not have sufficient information provided to allow any decision to be made and further information from the GP was requested.

26 referrals (43\%) were removed from CP onto RP at VLCC review: 14 did not require a CT; 12 scans were undertaken (7 high resolution CT, 1 CT pulmonary angiogram, 4 staging CT), 8 prior to clinic attendance.

34 referrals (57\%) remained on CP: 30 (88\%) proceeded to staging CT with average wait 12 days (range 3-17 days) from referral, all performed prior to clinic attendance. 1/34 died prior to clinic attendance. 3/34 were scanned before VLCC. A further 8 referrals were removed from CP after imaging.

Thus, only 36/60 (60\%) referrals were seen in the Lung Cancer Clinic. There was appropriate pathway change in $30 \%$ of referrals to General Respiratory (25\%) and Pleural Clinic (5\%).

Conclusion The VLCC can effectively assess and plan next investigation with appropriate clinic follow-up for suspected Lung Cancer patients. However, blind decision-making relies upon good clinical information from the referrer and administrative time can be wasted chasing this. Our data confirms that the VLCC facilitates efficient use of Out-patient and Radiology Services.
P86 OPTIMISING PATIENT FLOW AND USE OF RESOURCES IN THE TWO WEEK WAIT PATHWAY

K Goffe, P Ruparelia, CR Sander, C Butler. Cambridge University Hospitals, Cambridge, UK

10.1136/thoraxjnl-2016-209333.229

Introduction Lung cancer is the most common cause of cancer death in the UK. Survival is improving but is worse than in some European countries and North America. ${ }^{1}$ NICE guidelines recommend that patients with suspected malignancy are seen within two weeks of referral.

An earlier local audit found that $22 \%$ of patients referred on the pathway had lung cancer and identified a need to streamline the service. Referrals are triaged as high, intermediate and low risk by a respiratory Consultant based on chest X-ray and clinical details. High-risk patients are prioritised for CT imaging and lung clinical nurse specialist (CNS) time. All patients remain on the two week pathway regardless of triage status.

This study reviewed whether this triage system is accurate in identifying patients with malignancy, thereby improving resource utilisation.

Method Data was collected retrospectively on two week wait referrals during June and July 2015, using referral forms and electronic medical records. Data included key dates in the pathway, triage status and diagnosis.

Results 25 of 60 patients were triaged as high risk. Thirteen of these patients had cancer. Two patients with lung cancer were not triaged as high risk.

Triaging a patient as high risk had a sensitivity for lung malignancy of $86.7 \%$ and specificity of $71.4 \%$. Positive predictive value was 0.52 .

Nineteen patients triaged as high risk had a CT prior to clinician review and the remaining three had a CT within 3 days. This was three times higher than in the low/intermediate group.

The CNS attended the majority of initial clinic appointments in prioritised patients, unless CT showed benign disease.

Conclusion The triage method correctly identified patients with malignancy in the majority of cases. This led to efficient use of resources. Patients with lung cancer had earlier imaging and access to the CNS. Lung cancer symptoms can overlap with other respiratory conditions and following the initial clinical review, the respiratory clinician may decide to investigate some patients less urgently. In future, this method could help stratify urgency of referral.

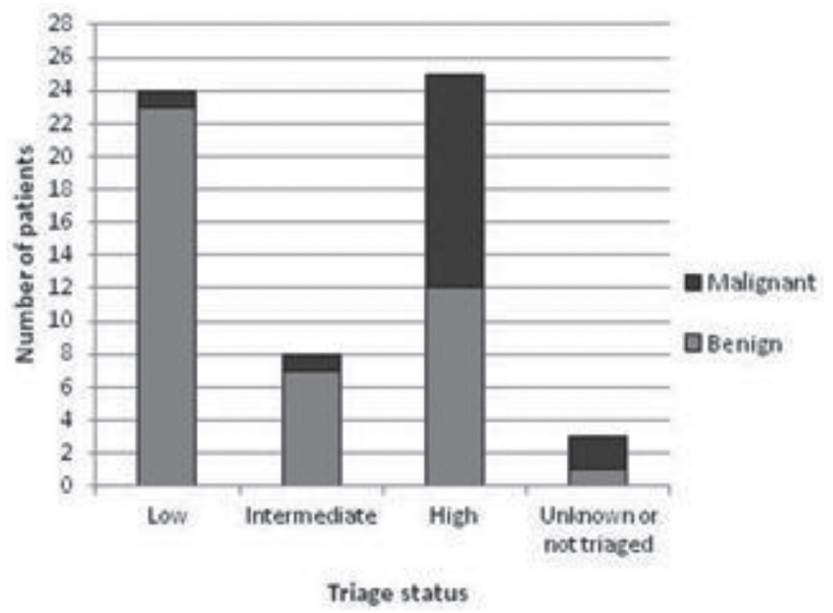

Abstract P86 Figure 1 Number of patients triages high/intermediate/ low with benign or malignant disease 